\title{
Cytokine (IL16) and tyrphostin actions on ovarian primordial follicle development
}

\author{
Amanda Feeney, Eric Nilsson and Michael K Skinner \\ School of Biological Sciences, Center for Reproductive Biology, Washington State University, Pullman, \\ Washington 99164-4236, USA \\ Correspondence should be addressed to M K Skinner; Email: skinner@wsu.edu
}

\begin{abstract}
An ovarian follicle is composed of an oocyte and surrounding theca and granulosa cells. Oocytes are stored in an arrested state within primordial follicles until they are signaled to re-initiate development by undergoing primordial-to-primary follicle transition. Previous gene bionetwork analyses of primordial follicle development identified a number of critical cytokine signaling pathways and genes potentially involved in the process. In the current study, candidate regulatory genes and pathways from the gene network analyses were tested for their effects on the formation of primordial follicles (follicle assembly) and on primordial follicle transition using whole ovary organ culture experiments. Observations indicate that the tyrphostin inhibitor (E)-2-benzylidene-3-(cyclohexylamino)-2,3-dihydro-1Hinden-1-one increased follicle assembly significantly, supporting a role for the MAPK signaling pathway in follicle assembly. The cytokine interleukin 16 (IL16) promotes primordial-to-primary follicle transition as compared with the controls, where as Delta-like ligand 4 (DLL4) and WNT-3A treatments have no effect. Immunohistochemical experiments demonstrated the localization of both the cytokine IL16 and its receptor CD4 in the granulosa cells surrounding each oocyte within the ovarian follicle. The tyrphostin LDN193189 (LDN) is an inhibitor of the bone morphogenic protein receptor 1 within the TGFB signaling pathway and was found to promote the primordial-to-primary follicle transition. Observations support the importance of cytokines (i.e., IL16) and cytokine signaling pathways in the regulation of early follicle development. Insights into regulatory factors affecting early primordial follicle development are provided that may associate with ovarian disease and translate to improved therapy in the future.

Reproduction (2014) 148 321-331
\end{abstract}

\section{Introduction}

The mammalian ovary is a dynamic organ in which germ cells go through many developmental stages to produce functional gametes. Development through these stages is initiated and regulated by different secreted growth factor and hormonal signals at different developmental points during oocyte maturation. During late fetal development in humans and early postnatal development in rodents, pre-granulosa cells and stromal mesenchymal cells surround the oogonia present in germ cell nests that then undergo follicle assembly to form primordial follicles (Skinner 2005; Fig. 1). A primordial follicle is composed of a single oocyte surrounded by a single layer of flattened squamous pre-granulosa cells (Skinner 2005). Primordial follicle assembly creates the pool of primordial follicles available during the reproductive life of the female. If selected for further development, the quiescent primordial follicles undergo the primordial-to-primary follicle transition. This is characterized by an increase in oocyte size and by proliferation of the cuboidal pre-granulosa cells that surround each oocyte (Fortune 2003, McLaughlin \& Mclver 2009, Kenngott et al. 2013;
Fig. 1B and C). Investigation of the cascade of molecular events involved in ovarian follicle development will provide insights into the etiology of certain ovarian diseases such as primary ovarian insufficiency (POI). $\mathrm{POI}$ is characterized by early depletion of the pool of arrested primordial follicles, and by induction of menopause before the age of 40 years (Coulam et al. 1986). POI affects $\sim 1 \%$ of women in the USA (Dixit et al. 2010). Understanding the molecular factors in early follicle development could lead to solutions for some infertilities in humans.

Previous studies have lead to the construction of proposed regulatory gene networks for primordial follicle assembly (Nilsson et al. 2013) and the primordial-to-primary follicle transition (Nilsson et al. 2010). In the current study, several signaling pathways and cytokine growth factors that were identified to potentially regulate primordial follicle development have been chosen to test experimentally. Follicle assembly has previously been shown to be regulated by several cytokines and growth factors that act in part by signaling through the MAPK signaling pathway. These include brain-derived neurotrophic factor 


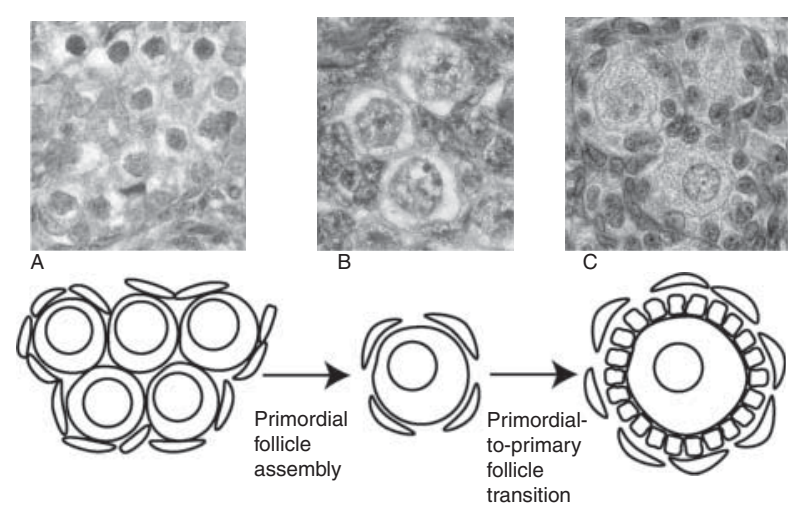

Figure 1 The process of primordial follicle development. (A) Oocyte nest, with surrounding pre-granulosa cells, (B) primordial follicles, and (C) primary follicles.

(Kerr et al. 2009), tumor necrosis factor $\alpha$ (Morrison \& Marcinkiewicz 2002, Nilsson et al. 2006a), and kit ligand (KITLG) (Wang \& Roy 2004). The MAPK and focal adhesion pathways have also been reported to be involved in gene networks previously proposed to regulate follicle assembly (Nilsson et al. 2013). Certain tyrphostin inhibitors (Levitzki 1996) are utilized in the current study to test whether the MAPK and focal adhesion pathways are involved in early ovarian follicle development. Tyrphostin inhibitor SP600125 inhibits c-Jun N-terminal kinase (JNK) in both the focal adhesion pathway and the MAPK signaling pathway. One would predict that this would lead to inhibited ovarian follicle assembly. (E)-2-benzylidene-3-(cyclohexylamino)-2,3dihydro-1 $\mathrm{H}$-inden-1-one $(\mathrm{BCl})$ is an allosteric inhibitor of MAPK phosphatases (MKPs), specifically dual-specificity phosphatase 6 (DUSP6), in the MAPK signaling pathway. DUSP6 is itself an inhibitor of ERK1/2 and JNK. $\mathrm{BCl}$ treatment can then be expected to stimulate ovarian follicle assembly.

The mRNA expression levels of interleukin 16 (IL 16), Wingless-type MMTV integration site family, member 2B (WNT2B), and Delta-like ligand 4 (DLL4) have been found to change during primordial-to-primary follicle transition (Nilsson et al. 2010). IL16 is a cytokine known to act as a chemoattractant for CD4 + T-cells in immune responses throughout the body (Kanehisa \& Goto 2000, Mathy et al. 2000, Cruikshank \& Little 2008). ILs have also been found in various ovarian cells including oocytes and supporting follicle cells in mammals, and have been shown to be secreted from macrophages in the ovary (Smolikova et al. 2012). Wnt2b is known to bind to the members of the Frizzled receptor family, which are a part of the Wnt signaling pathway. Wnt3a is also known to act on this same set of receptors (Kanehisa \& Goto 2000, Lapointe \& Boerboom 2011, Sirotkin 2011). The members of the Wnt signaling pathway have been found to be expressed in the ovary and to regulate follicle development and steroidogenesis (Richards \& Pangas 2010, Lapointe \& Boerboom 2011,
Sirotkin 2011). Dl/4 is known to bind to Notch receptors to activate the Notch signaling pathway (Kanehisa \& Goto 2000, Andrawes et al. 2013). Notch has been shown to influence multiple cellular processes including: cell fate decisions, proliferation, apoptosis, migration, and plasticity, and seems to be especially essential for angiogenesis (Thomas et al. 2013). In the current study, the roles of IL16, Wnt3a (as a functional substitute for Wnt $2 b$ which is not commercially available), and D//4 in primordial-to-primary follicle transition are investigated.

The roles of the MAPK and focal adhesion pathways on the primordial follicle transition are investigated in the current study using the inhibitors SP600125 and BCI. The role of the TGF $\beta$ signaling pathway is investigated using 4-(6-(4-(1-piperazinyl)phenyl]pyrazolo (1,5-a)pyrimidin-3-yl)-quinoline dihydrochloride (LDN193189). LDN193189 inhibits bone morphogenetic protein receptor 1 (BMPR1) in the TGFB signaling pathway. BMPR1 is a component of the receptor complex that anti-Müllerian hormone $(\mathrm{AMH})$ and bone morphogenetic proteins (BMP) bind (Nilsson et al. 2011). AMH is a protein hormone previously shown to have inhibitory action on both primordial follicle assembly (Nilsson et al. 2011) and primordial-to-primary follicle transition (Nilsson et al. 2007). Therefore LDN would be expected to stimulate the primordial follicle transition.

The current study investigates the actions of candidate regulatory factors and selected signaling pathways on ovarian follicle assembly and primordial-to-primary follicle transition. The objective is to test candidate regulatory pathways controlling follicle assembly with ovarian organ culture experiments using the tyrphostin inhibitors SP600125 and BCl. The candidate regulatory factors and pathways potentially controlling the primordial-to-primary follicle transition are tested using the cytokines IL16, DI/4, and Wnt3a, and tyrphostin inhibitors SP600125, BCl, and LDN193189. In addition, the localization of endogenous $I L 16$ and its receptor CD4 in ovarian follicles are determined using immunohistochemistry.

\section{Materials and methods}

\section{Organ culture and data collection}

Ovaries were removed from 0-day old Sprague-Dawley rat pups (for ovary follicle assembly studies) and 4-day-old rat pups (for ovary follicle transition studies). The animals were obtained from the Washington State University Center for Reproductive Biology Animal Core breeding colonies. All experimental protocols for the procedures with rats were pre-approved by the Washington State University Animal Care and Use Committee (IACUC approval \# 02568-026). These ovaries were then placed into a whole organ culture system as described previously (Nilsson et al. 2002, 2007, 2011). The whole organ culture system allows for modeling of multi-tissue biological processes. The culture medium contains $10 \mathrm{mg} / \mathrm{ml}$ gentamycin, 
40 units $/ \mathrm{ml}$ penicillin, and $10 \mathrm{mg} / \mathrm{ml}$ streptomycin. The microdissected ovaries were placed in groups of two or three in a culture well on a Teflon filter $(0.2 \mu \mathrm{m}$ Biopore membrane; Millipore Corp., Billerica, MA, USA) floating on a $500 \mu$ l culture medium (control) or a culture medium including specific treatments with IL16 (recombinant human, PeproTech cat\# 200-16A, Rocky Hill, NJ, USA) (20, 100, and $500 \mathrm{ng} / \mathrm{ml})$, DLL4 (R\&D Systems, cat\# 1389-D4, USA) (100 and $500 \mathrm{ng} / \mathrm{ml})$, WNT3A (R\&D Systems, cat\# 1324-WN, Minneapolis, MN, USA) (20 and $100 \mathrm{ng} / \mathrm{ml}$ ), SP600125 (LC Laboratories, cat\# S-7979, Woburn, MA, USA) (10 and $50 \mu \mathrm{M}), A M H$ (R\&D Systems, cat\# 1737-MS) (50 ng/ml), BCl (Sigma-Aldrich, cat\# B4313) $(0.5,1$ and $5 \mu \mathrm{M})$, or LDN193189 (Axon Medchem, cat\# 1509, Groningen, The Netherlands) $(0.003$ and $0.03 \mu \mathrm{g} / \mathrm{ml})$, along with positive controls KITLG (50 ng/ml) plus FGF2 (50 ng/ $\mathrm{ml}$ ). WNT3A was used as a functional substitute for $W N T 2 B$, as $W N T 2 B$ was not commercially available. Positive controls have been shown to affect follicle assembly or primordial follicle transition in previous studies (Nilsson et al. 2007, 2011). Ovaries were cultured for 2 days in the case of follicle assembly studies (Nilsson et al. 2011), and for 10 days in the case of primordialto-primary follicle transition studies (Nilsson et al. 2002). At the completion of the culture period, ovaries were processed as described previously (Nilsson et al. 2007, 2010, 2011).

After fixation, sectioning at $3 \mu \mathrm{M}$ thickness, and staining, the ovaries and follicles in each ovarian cross-section were categorized as either oocytes unassembled in nests, primordial follicles (stage 0), early primary i), primary ii), transitional iii), or pre-antral follicles iv) (Fig. 1B and C) as described previously (Nilsson et al. 2010, 2011). The oocytes and follicles from two adjacent sections of the largest cross-section of each ovary were counted and categorized, and then the counts in each category were averaged. In this way, two almost identical ovarian sections are evaluated, and an average number of unassembled oocytes, primordial follicles, and developing follicles are determined across those sections. Only oocytes with visible nuclei are counted. The total number of follicles was determined for each ovary cross section for each treatment, as well as the percent of follicles at each stage of development. These results were analyzed with ANOVA, Student's t-tests, and Dunnet's post hoc tests for specific comparisons.

\section{Immunohistochemistry}

Immunohistochemical procedures were carried out for IL16 and its known receptor CD4 in order to localize their expression in the developing ovary. Formalin-fixed tissues from 14-day-old and 19-day-old rat pups were used for immunohistochemical staining as described previously (Nilsson et al. 2002). The ovary sections were incubated with a blocking buffer (10\% goat serum (normal goat serum) or $10 \%$ rabbit serum (normal rabbit serum)), followed by the primary antibody, either anti-IL16 (Santa Cruz Biotechnology, Inc., cat\# sc-7902) (made in rabbit, $0.2,0.8$, or $4 \mu \mathrm{g} / \mathrm{ml}$ ) or anti-CD4 (Santa Cruz Biotechnology, Inc., cat\# sc-1140) (made in goat, $0.2,0.8$, or $4 \mu \mathrm{g} / \mathrm{ml}$ ) for antigen binding. Secondary antibody was biotinylated anti-rabbit IgG made in goat (Vector Laboratories, Inc., cat\# BA-1000, Burlingame, CA, USA) or biotinylated anti-goat IgG made in rabbit (Vector Laboratories,
Inc., cat\# BA-5000). The ovary sections were incubated with streptavidin-peroxidase Complex (Invitrogen, cat\# 50-420Z) for enzyme coupling. A color reaction was performed using a DAB peroxidase substrate kit (Vector Laboratories, Inc., cat\# SK-4100). Images were taken of the stained sections and the results were qualitatively analyzed.

\section{Results}

Ovarian organ culture experiments were used to investigate the potential role of candidate regulatory factors and selected signaling pathways on ovarian follicle assembly and/or primordial-to-primary follicle transition. For follicle assembly studies we used ovaries removed from 0-day-old rats and cultured for 2 days before being fixed and evaluated (Fig. 1). These 0-day-old rat ovaries contain predominately nests of oocytes not yet assembled into follicles (Nilsson et al. 2011). For primordial-to-primary follicle transition studies we used ovaries removed from 4-day-old rats and then cultured them for 10 days with replacement of organ culture medium every other day. These 4-day-old rat ovaries contain predominately assembled primordial follicles (Nilsson et al. 2007; Fig. 1). After culture and treatment, the ovaries were then fixed, sectioned, and evaluated for their proportions of unassembled oocytes, primordial follicles, and developing follicles as described in Materials and methods. The regulatory factors and pathways were selected for further investigation from previous system biology gene bionetwork studies (Nilsson et al. 2010, 2013).

\section{Primordial follicle assembly}

In order to test the importance of the MAPK signaling pathway to the follicle assembly process, 0-day-old ovaries were cultured for 2 days with or without the tyrphostin inhibitor compounds BCl and SP600125 (see Materials and methods). $\mathrm{BCl}$ acts indirectly to stimulate ERK1/2 and JNK. Both ERK and JNK play prominent roles in the MAPK pathway (Fig. 2). After 2 days of culture, untreated control ovaries had $26 \pm 3.0 \%$ assembled follicles. The ovaries treated with $\mathrm{BCl}(1 \mu \mathrm{M})$ had a significant increase in assembled follicles as compared with the control with $40 \pm 5.0 \%$ assembled into primordial follicles (Fig. 3A). There was no significant difference in the total oocyte count between control and $\mathrm{BCl}$-treated ovaries, indicating that oocyte death was not a confounding factor in these studies (Fig. 4B). These results indicate that stimulation of the MAPK pathway promotes the follicle assembly process.

The tyrphostin inhibitor SP600125 inhibits JNK in the MAPK and focal adhesion pathways (Fig. 2), and therefore decreases the activity of these pathways. Treatment of ovaries from 0-day-old rats with SP600125 did not have a significant effect on follicle assembly, as compared with the untreated controls. A $10 \mu \mathrm{M}$ SP600125 treatment resulted in $28 \pm 3.8 \%$ 


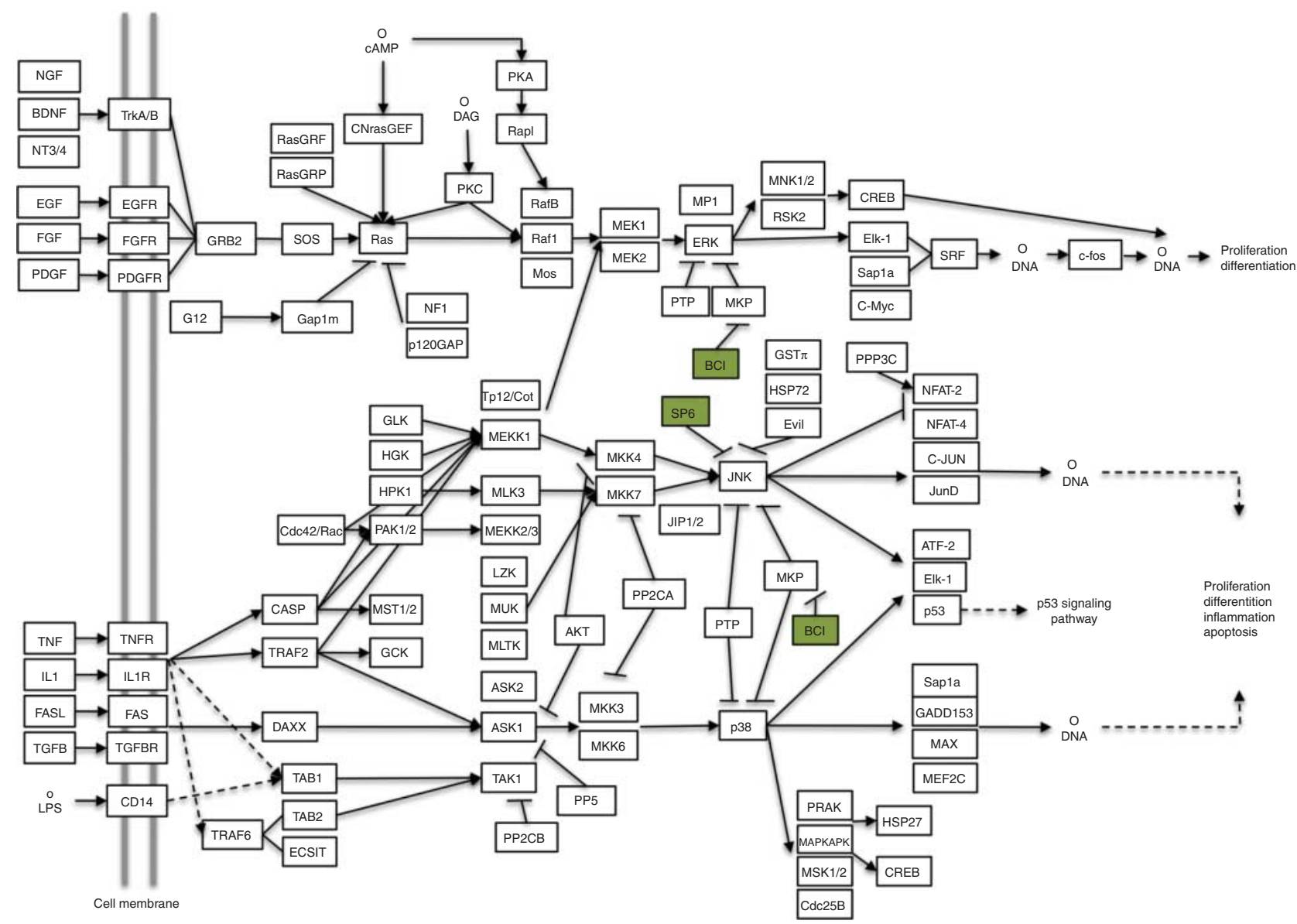

Figure 2 SP600125 and BCI inhibition in MAPK signaling pathway adapted from KEGG 04010. MAPK phosphatase (MKP) abbreviation. (www.genome.jp/kegg/pathway.html).

assembled follicles, while $50 \mu \mathrm{M}$ treatment with SP600125 resulted in $26 \pm 4.5 \%$ assembled follicles (Fig. 3A). This indicates that inhibition of the focal adhesion and MAPK pathways did not affect the follicle assembly process. This would also indicate that inhibition of JNK in the MAPK pathway has no effect on ovarian follicle assembly. However, treatment with SP600125 at $50 \mu \mathrm{M}$ concentration did result in a significant decrease in number of follicles per crosssection ( $31 \pm 7.2)$, as compared with controls ( $78 \pm 12.3$ ) (Fig. 3B). SP600125 treatment at $50 \mu \mathrm{M}$ was also observed to result in disorganized follicles with deteriorating cell nuclei (data not shown). This toxicity only occurs at the higher levels of the inhibitor. Together these observations indicate that increased MAPK signaling promoted follicle assembly, but that decreased MAPK and focal adhesion pathway signaling had little effect.

\section{Primordial-to-primary follicle transition}

A previous study suggests that the MAPK signaling pathway is a component of the gene network that regulates primordial follicle transition (Nilsson et al. 2010). In order to test this hypothesis, ovaries from 4-day-old rats were cultured for 10 days in the presence or absence of $\mathrm{BCl}$ or $\mathrm{SP} 600125$. $\mathrm{BCl}$ treatment resulted in no significant change in the proportion of developing follicles $(1 \mu \mathrm{M}: 64 \pm 4.8 \%, 5 \mu \mathrm{M}: 51 \pm 4.6 \%)$ as compared with the untreated controls $(58 \pm 2.4 \%)$ (Fig. 4A). There were also no significant differences in total follicle count between these treatment groups (Fig. 4B), indicating that oocyte death was not a confounding factor in these studies. Similarly, treatment with SP600125 resulted in no significant change in the proportion of developing follicles compared with the untreated controls (Fig. 4C). There was also no significant difference in total follicle count (Fig. 4D). These observations indicate that the MAPK and focal adhesion signaling pathways may not play prominent roles in the regulation of primordial-to-primary follicle transition, or that compensatory pathways exist. Therefore, growth factors known to affect follicle transition may act through additional parallel signaling pathways.

Previous research has shown that AMH produced by larger developing follicles will inhibit the primordialto-primary follicle transition (Visser \& Themmen 2005, Nilsson et al. 2007). Conversely, BMP4 and BMP7 are shown to stimulate primordial follicle transition (Lee et al. 

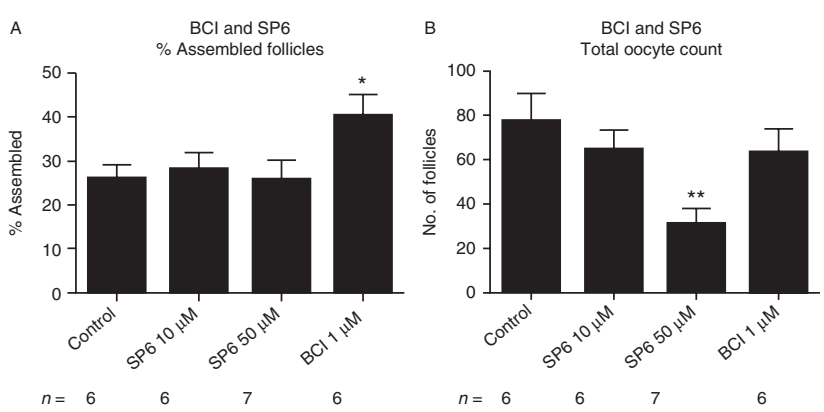

Figure 3 (A) Primordial follicle assembly after 2 days of treatment represented as \% assembled follicles with SP600125 and $\mathrm{BCl}$ treatments with statistical significance indicated as $* P<0.05$.

(B) Oocyte number per cross section after the same treatments. The mean \pm S.E.M. from a minimum of five different experiments carried out in replicate with statistical significance indicated as $* * P<0.01$.

2001, Nilsson \& Skinner 2003). $A M H$ and BMP growth factors can act through the BMPR1 branch of the TGFB family signaling pathway (Fig. 5). In the current study, ovaries were treated with the tyrphostin inhibitor LDN, which inhibits BMPRI, in order to determine how disruption of this TGFB family signaling pathway affects ovarian follicle development (Fig. 5). The control ovaries for these experiments were treated with dimethylsulfoxide vehicle. Control ovaries were found to have $46 \pm 2.2 \%$ developing follicles. LDN treatment at a concentration of $0.03 \mu \mathrm{g} / \mathrm{ml}$ resulted in a significant increase $(P<0.05)$ in the proportion of developing follicles, with $60 \pm 1.7 \%$ of the follicles in the developing stages. Treatment with LDN plus $50 \mathrm{ng} / \mathrm{ml} \mathrm{AMH}$ also resulted in a significant increase in developing follicles with $56 \pm 2.1 \%$ development (Fig. 6A). There were no significant differences between treatment groups in the total number of follicles per ovarian cross-section (Fig. 6B). The results for this treatment indicate that the BMP/AMH portion of the TGFB signaling pathway plays a role in primordialto-primary follicle transition, and that the inhibitory actions of $\mathrm{AMH}$ are blocked by LDN treatment.

Previous research had suggested that specific secreted growth factors and cytokines might have a role in the regulation of the primordial-to-primary follicle transition (Nilsson et al. 2010; Fig. 7). Three of these candidate regulatory factors, Wnt3a (as a functional substitute for Wnt2b), DI/4, and IL16 were chosen for evaluation. After the culture period, the untreated control ovaries had an average of $50 \pm 2.3 \%$ developing follicles. Neither Wnt3a nor DLL4 treatment resulted in any significant difference in the proportion of primordial to developing follicles as compared with the controls (Fig. 8A). There was also no significant difference in total follicle count between any of these treatment groups and the controls (Fig. 8B). In these experiments, positive control treatments with KITLG plus FGF2 were carried out, which stimulated primordialto-primary follicle transition, as expected. This indicates that experimental conditions supports follicle transition, even though WNT3A and DLL4 did not stimulate this.
The ovaries treated with $100 \mathrm{ng} / \mathrm{ml} / L 16$ had $57 \pm 1.4 \%$ developing follicles, which was a significant increase $(P<0.01)$ compared with the corresponding control group $(50 \pm 1.6 \%)$. The ovaries treated with $500 \mathrm{ng} / \mathrm{ml}$ IL16 had a mean of $58 \pm 1.9 \%$ developing follicles, which was also a significant increase compared with the control (Fig. 9A). IL 16 treatment at $20 \mathrm{ng} / \mathrm{ml}$ had no significant effect. None of the IL 16 treatments had a significant effect on the total counts of ovarian follicles as compared with the controls (Fig. 9B). These data suggest that the cytokine IL16 has a role in the regulation of the primordialto-primary ovarian follicle transition. This supports the importance of IL16 as part of a proposed network of genes regulating the primordial follicle transition (Fig. 7). As IL16 treatment had a significant effect on the primordialto-primary follicle transition, immunohistochemical experiments were carried out to localize IL16 and its receptor $C D 4$ within the ovary. Both $I L 16$ and $C D 4$ were found to be at highest levels in the granulosa cells of the developing follicle structures, including developing primordial follicles (Fig. 10). Negative controls using either nonspecific $\operatorname{lgG}$ as primary antibody, or using no primary antibody, showed no differential staining of granulosa cells compared with other ovarian cell types (Fig. 10). The observations suggest that IL16 is an active part of the network of genes regulating the primordial follicle transition, and that an apparent autocrine signaling loop between IL16 and CD4 is maintained in the granulosa cells of developing follicles.
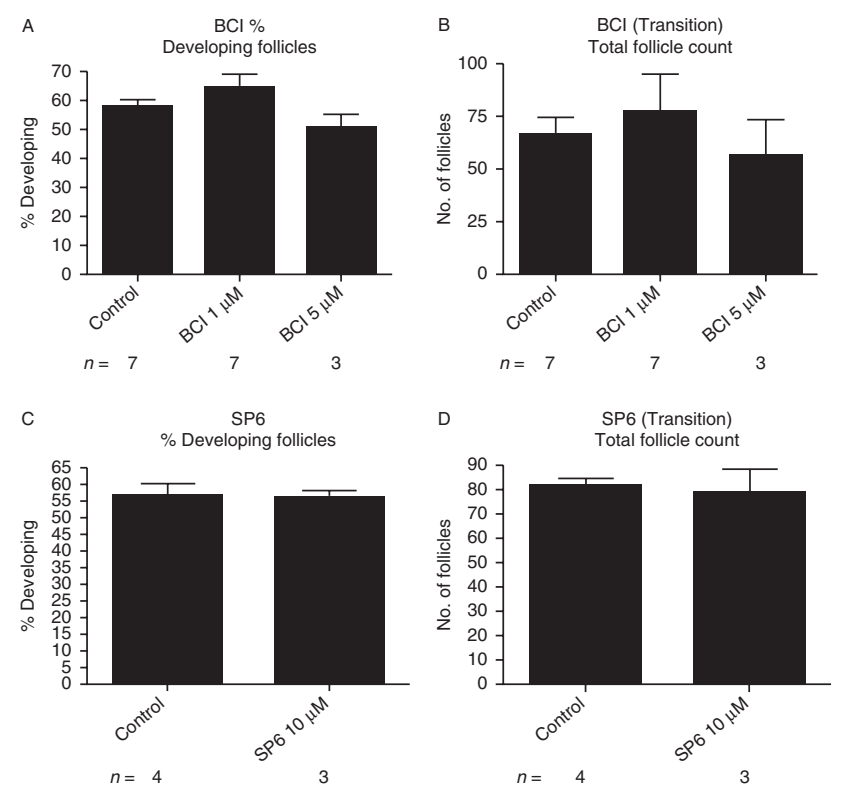

Figure 4 (A) Primordial-to-primary follicle transition after 10 days of treatment with $\mathrm{BCl}$ represented as \% developed follicles. (B) Oocyte number per cross section after the $\mathrm{BCl}$ treatments. The mean \pm s.E.M. from a minimum of three different experiments performed in replicate. (C) Primordial follicle transition after 10 days of treatment with SP600125 represented as \% developed follicles. (D) Oocyte number per cross section after SP600125 treatments. The mean \pm S.E.M. from a minimum of three different experiments carried out in replicate. 


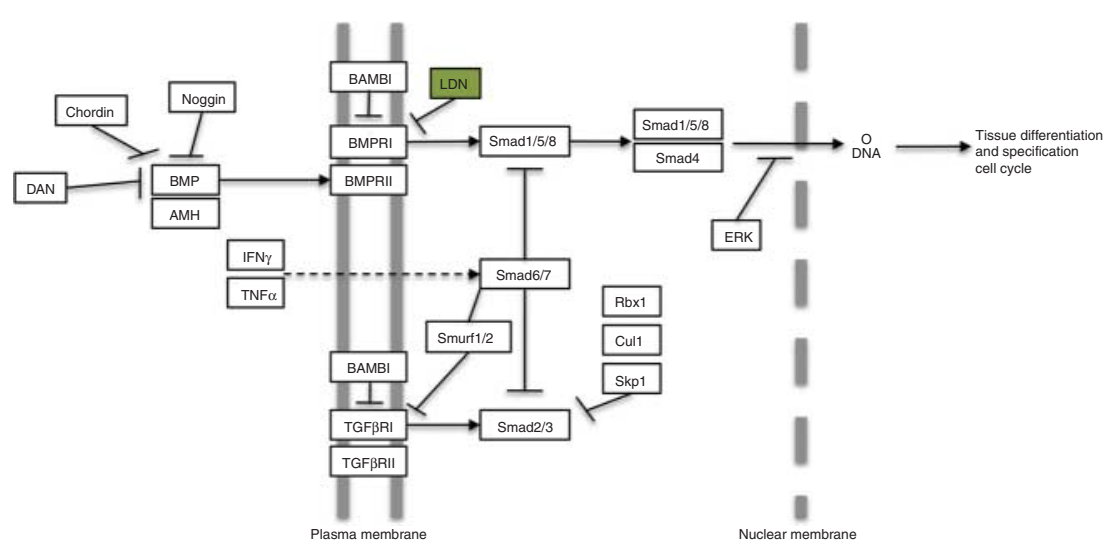

Figure 5 LDN inhibition in TGFB signaling pathway adapted from KEGG 04350. (www.genome.jp/kegg/pathway.html).

\section{Discussion}

Previous studies have used a systems biology approach to identify gene networks that potentially regulate primordial follicle assembly (Nilsson et al. 2013) and the primordialto-primary follicle transition (Nilsson et al. 2010). In these studies, the MAPK and focal adhesion signaling pathways were involved as mediating some of the actions of these proposed regulatory networks for both follicle assembly and the primordial follicle transition. In addition, examination of the proposed regulatory network for primordial follicle transition suggested that the TGFB signaling may be important, as well as WNT, Delta/Notch, and IL cytokine signaling. In the current study, these proposed regulatory gene networks are experimentally evaluated.

\section{Primordial follicle assembly}

In order to evaluate the roles of the MAPK and focal adhesion pathways in follicle assembly, 0-day-old rat ovaries were cultured and treated with the tyrphostin inhibitors $\mathrm{BCl}$ and SP600125. BCl has been shown to directly and specifically inactivate DUSP6 within the MAPK signaling pathway (Molina et al. 2009). DUSP6 is itself an inhibitor of ERK1/2 and JNK (Fig. 2), therefore the end result of $\mathrm{BCl}$ treatment is to increase MAPK activity. MAP kinases control many cellular events from embryogenesis, cell differentiation, cell proliferation, and cell death, to short-term changes required for homeostasis and acute hormonal responses (Chen et al. 2001). ERK1 and ERK2 are two specific MAP kinases that are coexpressed in all mammalian tissues and implicated as key regulators of cell proliferation and differentiation as well as oocyte maturation in culture (Su et al. 2002). The ovaries treated with $\mathrm{BCl}$ showed a significant increase in assembled follicles as compared with the controls. The observations indicate that increasing the activity of the MAPK pathway promotes the follicle assembly process (Fig. 11). This supports the previously proposed gene network for regulation of follicle assembly (Nilsson et al. 2013).
In order to test the effect of inhibiting the MAPK pathway during primordial follicle assembly these 0-day-old ovaries were cultured with SP600125. SP600125 is a small molecule inhibitor of JNK (Bennett et al. 2001), which plays a role in both the focal adhesion and MAPK signaling pathways. Both JNK and c-Jun have been shown to be essential for cell cycle progression and cell proliferation, and previous research has shown that inhibition of JNK via SP600125 halts in vitro growth of mouse preantral follicles (Oktem et al. 2011). In the present study, SP600125 treatment had no affect on the percentage of assembled ovarian follicles as compared with the controls. These results suggest that inhibition of JNK in either the focal adhesion pathway or the MAPK pathway does not have an effect on follicle assembly. Because of the significant decrease in total follicle number in ovaries treated with high concentrations of SP600125, the results also suggest that the SP600125 inhibitor has a toxic effect at higher concentrations. The ovaries treated with high concentrations of SP600125 have disorganized morphology and signs of necrotic cell death (data not shown).

An explanation is needed as to why $\mathrm{BCl}$ had an effect on follicle assembly via the MAPK pathway, whereas SP600125 did not. The inhibition of DUSP6 with $\mathrm{BCI}$
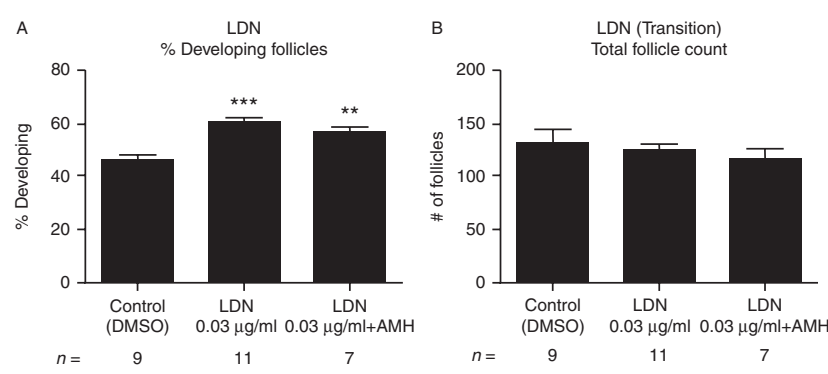

Figure 6 (A) Primordial follicle transition after 10 days of treatment represented as \% developing follicles after LDN, and LDN combined with $\mathrm{AMH}$ treatments with statistical significance indicated as $* * P<0.01$ and ${ }^{* * *} P<0.005$. (B) Oocyte number per cross section. The mean \pm s.E.M. from a minimum of five different experiments carried out in replicate. 


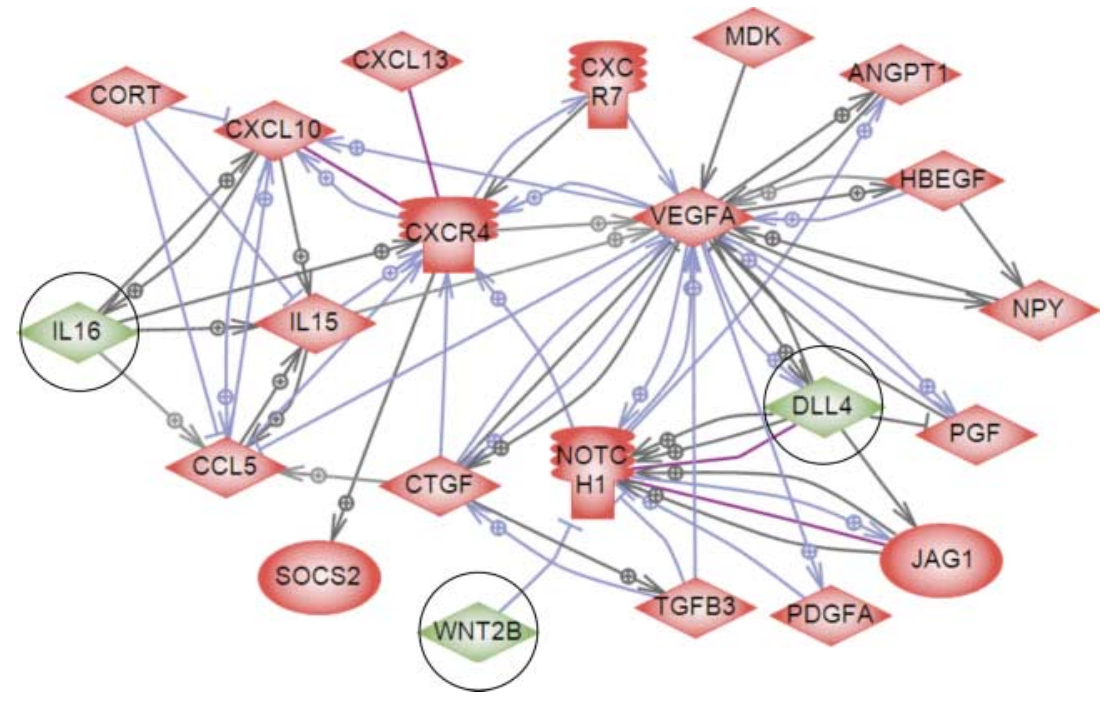

Figure 7 Scheme of direct connections between candidate regulatory growth factors and receptors previously found to be differentially expressed during primordial follicle transition. Circles around genes identify the candidate genes investigated. Nodes represent secreted extracellular signaling molecules and receptors that change in expression level during primordialto-primary follicle transition (except Notch1, which does not change in expression). Node shapes code: diamond - ligand; ice cream cone receptor. Green color represents the regulatory factors investigated in this paper. Connecting arrows represent relationships between genes present in published literature. Arrows with plus sign show positive regulation/activation, arrows with minus sign - negative regulation/inhibition, purple connections represent direct binding. Adapted from Nilsson et al. (2010). amplifies the proliferative effect of c-Jun and JNK signaling (Fig. 2). SP600125 decreases activity in this pathway by inhibiting JNK. This would mean that a decrease in activity in the MAPK pathway does not have an effect on follicle assembly, but an increase in activity at the JNK site does in fact affect development. This suggests that MAPK activity is sufficient to stimulate increased follicle assembly, but that the activity of the pathway is not necessary for follicle assembly to occur, perhaps because of compensation with other signaling pathways. Further investigation is needed to clarify the molecular mechanisms underlying the differences in response to $\mathrm{BCl}$ and SP600125 treatments.

\section{Primordial-to-primary follicle transition}

A previous systems biology investigation showed that mRNA expression levels of the secreted growth factors Wnt2b, DI/4, and IL16 changed in ovaries during primordial-to-primary follicle transition (Fig. 7; Nilsson et al. 2010). In the current study, organ culture experiments were carried out to directly test whether these signaling molecules regulate follicle transition. Wnt3a acts on the same receptor as $\mathrm{Wnt} 2 \mathrm{~b}$ and is assumed to have the same physiological effects; therefore Wnt3a was used as a functional substitute treatment factor to determine effects on ovarian follicle primordial to primary transition. The WNT2B is not commercially available. In this study, Wnt3a had no significant effect on the primordialto-primary follicle transition as compared with the controls. Even though $W n t 2 b$ is known to be present in the ovary, it has been shown that when the $W n t 2 b$ gene was knocked out in mice that were viable, fertile, and had a normal reproductive lifespan (Tsukiyama \& Yamaguchi 2012). This indicates that Wnt2b is not essential for follicle transition despite its presence in the ovary and/or that compensatory factors exist. A previous study suggests that Wnt2b mRNA expression in the ovary is localized to the rat ovarian surface epithelium (Ricken etal. 2002). This would support the idea that Wnt2b may have an indirect supporting role within the ovary with regard to follicle development.

DLL4 is known to bind to Notch receptors to activate the Notch signaling pathway (Musse et al. 2012). Notch has been shown to influence multiple cellular processes including: cell fate decisions, proliferation, apoptosis, migration, and plasticity, and seems to be especially essential for processes such as angiogenesis (Thomas et al. 2013). In a previous study, D//4 was shown to be expressed in ovaries (Nilsson et al. 2010), but in this study DLL4 treatment resulted in no significant changes in the percentage of follicle transition as compared with the controls (Fig. 8). This may indicate a nonessential supporting role of DLL4 within the ovary. Alternatively, Dll4 expression at the time of follicle transition may prepare the way for vascular development that occurs later as follicle develop. In a previous study, loss of D/l4 expression or Notch signaling has lead to abnormal development of the vascular network, but was not shown to be directly related to the process of angiogenesis (Mettouchi 2012).

IL16 is a cytokine often associated with immune response, especially for immunoregulatory responses (Mahindra \& Anderson 2012). IL16 is produced by CD8positive T cells and its known receptor is the $C D 4$ molecule (Cruikshank et al. 1996). A previous study showed that mRNA expression levels of IL 16 changed in ovaries during primordial-to-primary follicle transition (Nilsson et al. 2010; Fig. 7). Based on these data, organ culture experiments were conducted to test the activity of IL16 within the ovary, and immunohistochemical experiments were then carried out to specifically locate both IL16 and 

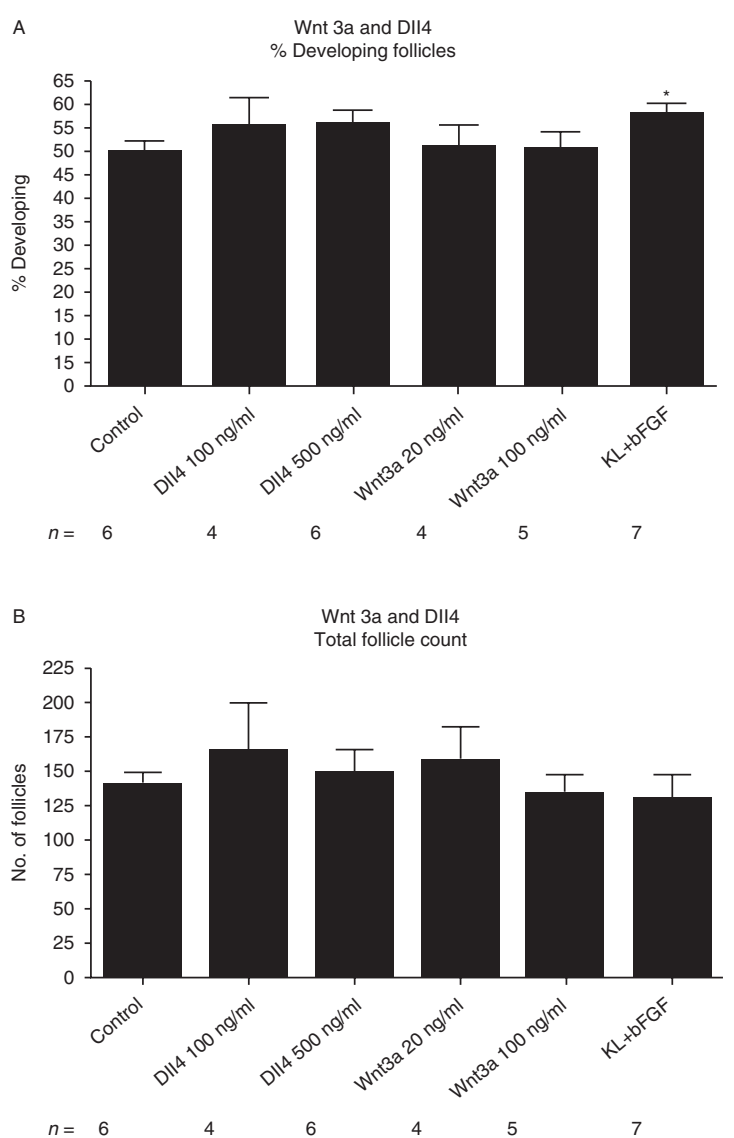

Figure 8 (A) Primordial follicle transition after 10 days of treatment represented as \% developed follicles after WNT3A, DLL4, and KL+ BFGF treatments with statistical significance indicated as $* P<0.05$. (B) Oocyte number per cross section. The mean \pm S.E.M. from a minimum of three different experiments carried out in replicate.

its receptor CD4 in developing ovaries. The organ culture experiments demonstrated a moderate increase in primordial-to-primary follicle transition as compared with the controls. The immunohistochemical experiments showed that both IL16 and CD4 proteins were located within the granulosa cells of ovarian follicles, suggesting an autocrine signaling loop. Therefore, IL16 appears to promote the primordial follicle transition process (Fig. 11).

A systems biology investigation of the primordialto-primary follicle transition predicted that MAPK signaling was involved in this process (Nilsson et al. 2010). The importance of MAPK signaling in the regulation of the primordial-to-primary follicle transition was investigated in the current studies by using the tyrphostin inhibitors $\mathrm{BCl}$ and SP600125 to treat 4-day-old rat ovaries cultured for 10 days. The ovaries treated with $\mathrm{BCl}$ at this stage of development showed no significant difference compared with the controls. This suggests that stimulating the MAPK pathway may not be an important mode of regulating the ovarian primordial-to-primary follicle transition, even though it did seem to have an effect earlier in the developmental process. The ovaries treated with
SP600125 at this stage also showed no significant difference between untreated controls and the treated ovaries, at either treatment concentration. This indicates that neither directly inhibiting JNK within the MAPK pathway nor inhibiting the focal adhesion pathway affects development at this stage and therefore neither is vital for regulating the primordial follicle transition. Several growth factors that can act in part by signaling through the MAPK pathway have been shown to regulate the primordial-to-primary follicle transition, including PDGF (Nilsson et al. 2006b), FGF2 (Nilsson et al. 2001, Jin et al. 2005, Chaves et al. 2012), NTF3 (Nilsson et al. 2009), and KITLD (Parrott \& Skinner 1999). Therefore, these factors likely affect follicle transition by acting through other parallel signaling pathways, rather than MAPK exclusively. C-erbB2 signaling has been shown to promote primordial follicle development in rats, and this increased follicle transition was blocked by treatment with the MAPK inhibitor PD98059 (Li-Ping et al. 2010), suggesting an active role for MAPK signaling in this process. In addition, IL16 signaling through the CD4 receptor has been shown to activate MAP kinases in immune B-cells, as well as NFkB and P27 signaling pathways (Yang et al. 2013). A cross-talk between several intracellular pathways is likely when regulating the primordial-to-primary follicle transition, including MAPK, NFkB, PI3K, and PTEN (Jin et al. 2005, Serafica et al. 2005, Liu et al. 2006, Jagarlamudi et al. 2009, Adhikari et al. 2013, Morohaku et al. 2013). Therefore, the signaling that occurs within the MAPK pathway may have effects that are distributed across other pathways, such as PI3K. It is possible that inhibiting JNK with SP600125 allows cross-talk to other pathways and therefore has little effect on follicle transition, while inhibiting MEK in the MAPK pathway with PD98059 does slow follicle transition when ovaries from 2-day-old rats are treated. In the current experiments, it has been demonstrated that a completely intact MAPK signaling pathway, including fully functional JNK, is not necessary for the primordial-to-primary follicle
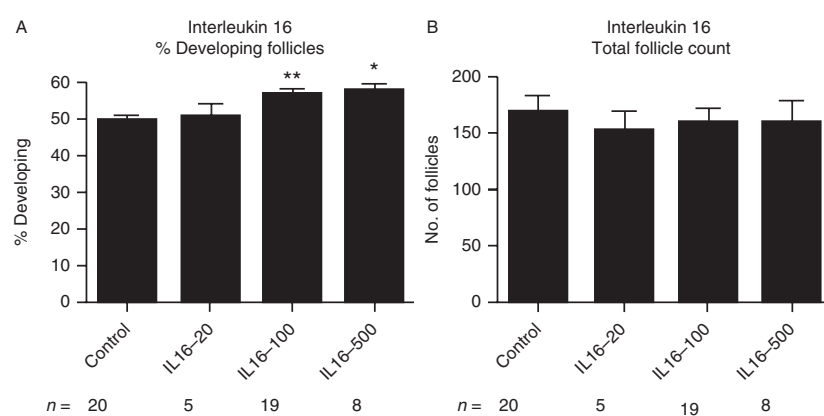

Figure 9 (A) Primordial follicle transition after 10 days of IL16 treatment represented as \% developed follicles. Statistical significance indicated as $* P<0.05$ and ${ }^{* *} P<0.01$. (B) Oocyte number per cross section after the same treatments. The mean \pm s.E.M. from a minimum of five different experiments carried out in replicate. 


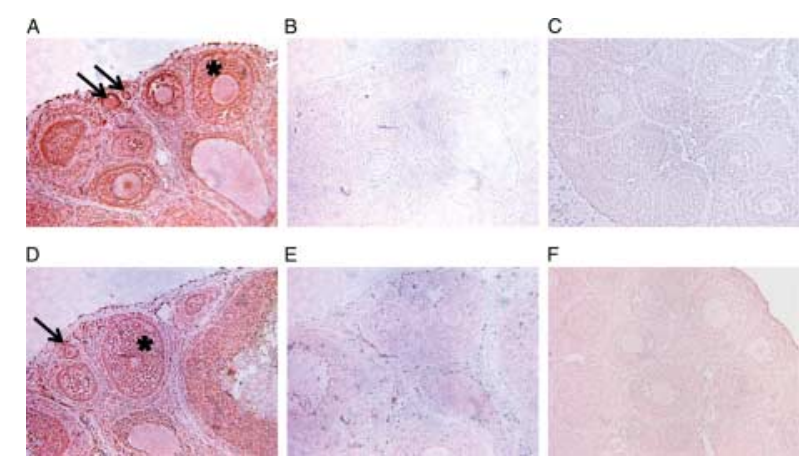

Figure 10 Immunohistochemical analysis of interleukin 16 and its receptor, CD4. Brown stain indicates presence of the specified protein. (A) Localization of IL16 in the ovary, particularly in granulosa cells of follicles $(*)$. (B) Negative control experiment conducted without the use of primary antibody. (C) Negative control experiment conducted with nonspecific rabbit IgG. (D) Localization of CD4 receptor in the ovary, particularly in granulosa cells of follicles $(*)$. (E) Negative control experiment conducted without the use of primary antibody.

(F) Negative control experiment conducted with nonspecific goat IgG. Arrows point to developing primordial and primary follicles.

transition. Increasing MAPK pathway activity with $\mathrm{BCl}$ also did not result in increased follicle transition. Therefore, IL16 treatment appears to increase follicle transition through additional parallel signaling pathways, rather than MAPK alone.

A previous study suggested that TGFB pathway signaling was another mechanism regulating the primordial-to-primary follicle transition (Nilsson et al. 2010). In order to determine whether disruption of a specific branch of the TGFB signaling pathway affects primordial follicle transition, a third tyrphostin inhibitor was selected for use in organ culture experiments. The tyrphostin inhibitor LDN inhibits BMPRI within the TGFB signaling cascade (Cuny et al. 2008; Fig. 5). BMPs induce many different cellular effects ranging from stem cell maintenance, migration, differentiation, and proliferation to apoptosis depending on context (Sieber et al. 2009). AMH also binds to BMPR1 (Cuny et al. 2008), and has been shown in previous studies to inhibit primordial follicle assembly and transition (Durlinger et al. 1999, Visser \& Themmen 2005, Nilsson et al. 2007, 2011). In the current study, LDN was shown to have a significant effect on ovarian primordial-to-primary follicle transition. These previous observations suggest that LDN treatment would increase the percentage of developing follicles. This was verified by the results of this study. When combined with $\mathrm{AMH}$, there was still a significant increase in the percentage of primary follicles as compared with the controls, likely indicating LDN is blocking the activity of $\mathrm{AMH}$ at the receptor. Together, these data indicate that signaling through the BMPR1 branch of the TGFB pathway regulates the primordialto-primary follicle transition (Fig. 11). This is consistent with previous studies showing that the growth factors $\mathrm{AMH}$ and BMP4 affect primordial follicle transition
(Nilsson \& Skinner 2003, Nilsson et al. 2007, van Houten et al. 2010, Buratini \& Price 2011) and helps to validate the predictions of previous systems biology investigations (Nilsson et al. 2010) that suggested the TGFB signaling pathway helped to regulate this process.

\section{Conclusions}

In summary, specific signaling networks and candidate regulatory factors were tested for their roles in the regulation of early follicle development. Based on inhibitor experimentation in this study, the MAPK signaling pathway was shown to be important for the regulation of primordial follicle assembly. IL16 has now been shown to play an active role in the regulation of transition from primordial follicles with arrested oocytes into primary follicles with developing oocytes and proliferating granulosa cells. IL16 and its receptor CD4 were also specifically localized to the granulosa cells of developing follicles. Both Wnt2b and DLL4 treatment could have indirect roles with regard to primordial follicle transition within the ovary, but while mRNA expression of these cytokines was regulated within the ovary (Nilsson et al. 2010; Fig. 7), neither Wnt2b nor DLL4 treatment had a direct effect on primordial-to-primary follicle transition in cultured ovaries. The TGFB signaling pathway through BMPR1 was shown to be important for regulating primordial-to-primary follicle transition. Therefore IL16, the MAPK pathway, and the TGFB pathway were experimentally confirmed to be a part of the network of signaling molecules and pathways that regulate early ovarian follicle development (Fig. 11).

The results of these studies support the utility of the previously proposed gene networks in the identification of candidate regulatory points to control follicle development (Nilsson et al. 2010, 2011). A systems biology approach to study the developmental processes can be a tool that is complementary to the more reductionist experiments, such as performed in the current investigation. Some findings from this study supported the predictions from the systems biology investigations and some did not. This emphasizes the importance of using both experimental approaches.

Oocyte maturation and follicle development are complicated processes and the pathways and factors

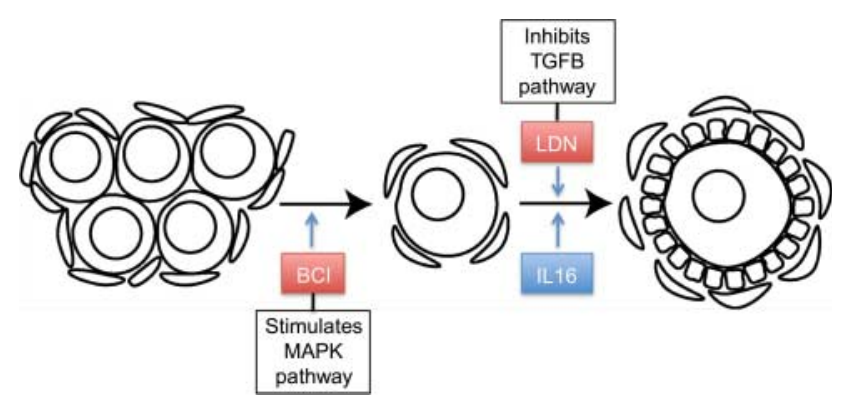

Figure 11 Primordial follicle developmental summary. 
regulating primordial follicle assembly and primordialto-primary follicle transition are still being determined. Understanding the regulators of early follicle development could lead to solutions for certain types of infertility in mammals, including humans. POI leading to early menopause (Dixit et al. 2010) is increasingly becoming a detriment to female reproductive success. Further investigation of the cytokine and hormonal signaling cascades involved in ovarian development will help to advance and improve treatments to combat ovarian disease.

\section{Declaration of interest}

The authors declare that there is no conflict of interest that could be perceived as prejudicing the impartiality of the research reported.

\section{Funding}

This research was supported by NIH grants to M K Skinner.

\section{Acknowledgements}

The authors acknowledge the technical assistance of Ms Alison Durand and Ms Heather Johnson for assistance in preparation of the manuscript.

\section{References}

Adhikari D, Risal S, Liu K \& Shen Y 2013 Pharmacological inhibition of mTORC1 prevents over-activation of the primordial follicle pool in response to elevated PI3K signaling. PLoS ONE 8 e53810. (doi:10.1371/ journal.pone.0053810)

Andrawes MB, Xu X, Liu H, Ficarro SB, Marto JA, Aster JC \& Blacklow SC 2013 Intrinsic selectivity of Notch 1 for Delta-like 4 over Delta-like 1. Journal of Biological Chemistry 288 25477-25489. (doi:10.1074/jbc. M113.454850)

Bennett BL, Sasaki DT, Murray BW, O'Leary EC, Sakata ST, Xu W, Leisten JC, Motiwala A, Pierce S, Satoh Y et al. 2001 SP600125, an anthrapyrazolone inhibitor of Jun N-terminal kinase. PNAS 98 13681-13686. (doi:10.1073/pnas.251194298)

Buratini J \& Price CA 2011 Follicular somatic cell factors and follicle development. Reproduction, Fertility, and Development 23 32-39. (doi:10.1071/RD10224)

Chaves RN, de Matos MH, Buratini J Jr \& de Figueiredo JR 2012 The fibroblast growth factor family: involvement in the regulation of folliculogenesis. Reproduction, Fertility, and Development $\mathbf{2 4}$ 905-915. (doi:10.1071/RD11318)

Chen Z, Gibson TB, Robinson F, Silvestro L, Pearson G, Xu B, Wright A, Vanderbilt C \& Cobb MH 2001 MAP kinases. Chemical Reviews 101 2449-2476. (doi:10.1021/cr000241p)

Coulam CB, Adamson SC \& Annegers JF 1986 Incidence of premature ovarian failure. Obstetrics and Gynecology 67 604-606.

Cruikshank W \& Little F 2008 Interleukin-16: the ins and outs of regulating T-cell activation. Critical Reviews in Immunology 28 467-483. (doi:10. 1615/CritRevImmunol.v28.i6.10)

Cruikshank W, Kornfeld H, Berman J, Chupp G, Keane J \& Center D 1996 Biological activity of interleukin-16. Nature 382 501-502. (doi:10.1038/ 382501b0)

Cuny GD, Yu PB, Laha JK, Xing X, Liu JF, Lai CS, Deng DY, Sachidanandan C, Bloch KD \& Peterson RT 2008 Structure-activity relationship study of bone morphogenetic protein (BMP) signaling inhibitors. Bioorganic \& Medicinal Chemistry Letters 18 4388-4392. (doi:10.1016/j.bmcl.2008.06.052)
Dixit H, Rao L, Padmalatha V, Raseswari T, Kapu AK, Panda B, Murthy K, Tosh D, Nallari P, Deenadayal M et al. 2010 Genes governing premature ovarian failure. Reproductive Biomedicine Online 20 724-740. (doi:10. 1016/j.rbmo.2010.02.018)

Durlinger AL, Kramer P, Karels B, de Jong FH, Uilenbroek JT, Grootegoed JA \& Themmen AP 1999 Control of primordial follicle recruitment by anti-Müllerian hormone in the mouse ovary. Endocrinology 140 5789-5796. (doi:10.1210/endo.140.12.7204)

Fortune JE 2003 The early stages of follicular development: activation of primordial follicles and growth of preantral follicles. Animal Reproduction Science 78 135-163. (doi:10.1016/S0378-4320(03)00088-5)

van Houten EL, Themmen AP \& Visser JA 2010 Anti-Müllerian hormone (AMH): regulator and marker of ovarian function. Annales d'Endocrinologie 71 191-197. (doi:10.1016/j.ando.2010.02.016)

Jagarlamudi K, Liu L, Adhikari D, Reddy P, Idahl A, Ottander U, Lundin E \& Liu K 2009 Oocyte-specific deletion of Pten in mice reveals a stage-specific function of PTEN/PI3K signaling in oocytes in controlling follicular activation. PLoS ONE 4 e6186. (doi:10.1371/journal.pone.0006186)

Jin X, Han CS, Zhang XS, Yuan JX, Hu ZY \& Liu YX 2005 Signal transduction of stem cell factor in promoting early follicle development. Molecular and Cellular Endocrinology 229 3-10. (doi:10.1016/j.mce. 2004.10.006)

Kanehisa M \& Goto S 2000 KEGG: kyoto encyclopedia of genes and genomes. Nucleic Acids Research 28 27-30. (doi:10.1093/nar/28.1.27)

Kenngott RA, Vermehren M, Ebach K \& Sinowatz F 2013 The role of ovarian surface epithelium in folliculogenesis during fetal development of the bovine ovary: a histological and immunohistochemical study. Sexual Development 7 180-195. (doi:10.1159/000348881)

Kerr B, Garcia-Rudaz C, Dorfman M, Paredes A \& Ojeda SR 2009 NTRK1 and NTRK2 receptors facilitate follicle assembly and early follicular development in the mouse ovary. Reproduction 138 131-140. (doi:10. 1530/REP-08-0474)

Lapointe E \& Boerboom D 2011 WNT signaling and the regulation of ovarian steroidogenesis. Frontiers in Bioscience 3 276-285. (doi:10. 2741/s151)

Lee WS, Otsuka F, Moore RK \& Shimasaki S 2001 Effect of bone morphogenetic protein-7 on folliculogenesis and ovulation in the rat. Biology of Reproduction 65 994-999. (doi:10.1095/biolreprod65.4.994)

Levitzki A 1996 Targeting signal transduction for disease therapy. Current Opinion in Cell Biology 8 239-244. (doi:10.1016/S09550674(96)80071-8)

Li-Ping Z, Da-Lei Z, Jian H, Liang-Quan X, Ai-Xia X, Xiao-Yu D, Dan-Feng T \& Yue-Hui Z 2010 Proto-oncogene c-erbB2 initiates rat primordial follicle growth via PKC and MAPK pathways. Reproductive Biology and Endocrinology 8 66. (doi:10.1186/1477-7827-8-66)

Liu K, Rajareddy S, Liu L, Jagarlamudi K, Boman K, Selstam G \& Reddy P 2006 Control of mammalian oocyte growth and early follicular development by the oocyte PI3 kinase pathway: new roles for an old timer. Developmental Biology 299 1-11. (doi:10.1016/j.ydbio.2006.07.038)

Mahindra A \& Anderson KC 2012 Role of interleukin 16 in multiple myeloma pathogenesis: a potential novel therapeutic target? Journal of the National Cancer Institute 104 964-965. (doi:10.1016/j.ydbio.2006.07.038)

Mathy NL, Scheuer W, Lanzendorfer M, Honold K, Ambrosius D, Norley S \& Kurth R 2000 Interleukin-16 stimulates the expression and production of pro-inflammatory cytokines by human monocytes. Immunology $\mathbf{1 0 0}$ 63-69. (doi:10.1046/j.1365-2567.2000.00997.x)

McLaughlin EA \& Mclver SC 2009 Awakening the oocyte: controlling primordial follicle development. Reproduction 137 1-11. (doi:10.1530/ REP-08-0118)

Mettouchi A 2012 The role of extracellular matrix in vascular branching morphogenesis. Cell Adhesion \& Migration 6 528-534. (doi:10.4161/ cam.22862)

Molina G, Vogt A, Bakan A, Dai W, Queiroz de Oliveira P, Znosko W, Smithgall TE, Bahar I, Lazo JS, Day BW et al. 2009 Zebrafish chemical screening reveals an inhibitor of Dusp6 that expands cardiac cell lineages. Nature Chemical Biology 5 680-687. (doi:10. 1038/nchembio.190)

Morohaku K, Hoshino Y, Sasada H \& Sato E 2013 Incorporation of phosphatase inhibitor in culture prompts growth initiation of isolated non-growing oocytes. PLOS ONE 8 e77533. (doi:10.1371/journal.pone. 0077533) 
Morrison LJ \& Marcinkiewicz JL 2002 Tumor necrosis factor $\boldsymbol{\alpha}$ enhances oocyte/follicle apoptosis in the neonatal rat ovary. Biology of Reproduction 66 450-457. (doi:10.1095/biolreprod66.2.450)

Musse AA, Meloty-Kapella L \& Weinmaster G 2012 Notch ligand endocytosis: mechanistic basis of signaling activity. Seminars in Cell \& Developmental Biology 23 429-436. (doi:10.1016/j.semcdb.2012.01.011)

Nilsson EE \& Skinner MK 2003 Bone morphogenetic protein-4 acts as an ovarian follicle survival factor and promotes primordial follicle development. Biology of Reproduction 69 1265-1272. (doi:10.1095/ biolreprod.103.018671)

Nilsson E, Parrott JA \& Skinner MK 2001 Basic fibroblast growth factor induces primordial follicle development and initiates folliculogenesis. Molecular and Cellular Endocrinology 175 123-130. (doi:10.1016/ S0303-7207(01)00391-4)

Nilsson EE, Kezele P \& Skinner MK 2002 Leukemia inhibitory factor (LIF) promotes the primordial to primary follicle transition in rat ovaries. Molecular and Cellular Endocrinology 188 65-73. (doi:10.1016/S03037207(01)00746-8)

Nilsson EE, Stanfield J \& Skinner MK 2006a Interactions between progesterone and tumor necrosis factor- $\alpha$ in the regulation of primordial follicle assembly. Reproduction 132 877-886. (doi:10.1530/REP-06-0045)

Nilsson EE, Detzel C \& Skinner MK 2006b Platelet-derived growth factor modulates the primordial to primary follicle transition. Reproduction 131 1007-1015. (doi:10.1530/rep.1.00978)

Nilsson E, Rogers N \& Skinner MK 2007 Actions of anti-Müllerian hormone on the ovarian transcriptome to inhibit primordial to primary follicle transition. Reproduction 134 209-221. (doi:10.1530/REP-07-0119)

Nilsson E, Dole G \& Skinner MK 2009 Neurotrophin NT3 promotes ovarian primordial to primary follicle transition. Reproduction 138 697-707. (doi:10.1530/REP-09-0179)

Nilsson EE, Savenkova MI, Schindler R, Zhang B, Schadt EE \& Skinner MK 2010 Gene bionetwork analysis of ovarian primordial follicle development. PLoS ONE 5 e11637. (doi:10.1371/journal.pone.0011637)

Nilsson EE, Schindler R, Savenkova MI \& Skinner MK 2011 Inhibitory actions of anti-Müllerian hormone $(\mathrm{AMH})$ on ovarian primordial follicle assembly. PLoS ONE 6 e20087. (doi:10.1371/journal.pone.0020087)

Nilsson E, Zhang B \& Skinner MK 2013 Gene bionetworks that regulate ovarian primordial follicle assembly. BMC Genomics 14496. (doi:10.1186/1471-2164-14-496)

Oktem O, Buyuk E \& Oktay K 2011 Preantral follicle growth is regulated by c-Jun-N-terminal kinase (JNK) pathway. Reproductive Sciences 18 269-276. (doi:10.1177/1933719110385709)

Parrott JA \& Skinner MK 1999 Kit-ligand/stem cell factor induces primordial follicle development and initiates folliculogenesis. Endocrinology 140 4262-4271. (doi:10.1210/endo.140.9.6994)

Richards JS \& Pangas SA 2010 The ovary: basic biology and clinical implications. Journal of Clinical Investigation 120 963-972. (doi:10.1172/ JCl41350)
Ricken A, Lochhead P, Kontogiannea M \& Farookhi R 2002 Wnt signaling in the ovary: identification and compartmentalized expression of wnt-2, wnt2b, and frizzled-4 mRNAs. Endocrinology 143 2741-2749. (doi:10.1210/ endo.143.7.8908)

Serafica MD, Goto T \& Trounson AO 2005 Transcripts from a human primordial follicle cDNA library. Human Reproduction 20 2074-2091. (doi:10.1093/humrep/dei030)

Sieber C, Kopf J, Hiepen C \& Knaus P 2009 Recent advances in BMP receptor signaling. Cytokine \& Growth Factor Reviews 20 343-355. (doi:10.1016/j.cytogfr.2009.10.007)

Sirotkin AV 2011 Growth factors controlling ovarian functions. Journal of Cellular Physiology 226 2222-2225. (doi:10.1002/jcp.22588)

Skinner MK 2005 Regulation of primordial follicle assembly and development. Human Reproduction Update 11 461-471. (doi:10.1093/ humupd/dmi020)

Smolikova K, Mlynarcikova A \& Scsukova S 2012 Role of interleukins in the regulation of ovarian functions. Endocrine Regulations 46 237-253. (doi:10.4149/endo_2012_04_237)

Su YQ, Wigglesworth K, Pendola FL, O'Brien MJ \& Eppig JJ 2002 Mitogenactivated protein kinase activity in cumulus cells is essential for gonadotropin-induced oocyte meiotic resumption and cumulus expansion in the mouse. Endocrinology 143 2221-2232. (doi:10.1210/ endo.143.6.8845)

Thomas JL, Baker K, Han J, Calvo C, Nurmi H, Eichmann AC \& Alitalo K 2013 Interactions between VEGFR and Notch signaling pathways in endothelial and neural cells. Cellular and Molecular Life Sciences 70 1779-1792. (doi:10.1007/s00018-013-1312-6)

Tsukiyama T \& Yamaguchi TP 2012 Mice lacking Wnt2b are viable and display a postnatal olfactory bulb phenotype. Neuroscience Letters 512 48-52. (doi:10.1016/j.neulet.2012.01.062)

Visser JA \& Themmen AP 2005 Anti-Müllerian hormone and folliculogenesis. Molecular and Cellular Endocrinology 234 81-86. (doi:10.1016/ j.mce.2004.09.008)

Wang J \& Roy SK 2004 Growth differentiation factor-9 and stem cell factor promote primordial follicle formation in the hamster: modulation by follicle-stimulating hormone. Biology of Reproduction 70 577-585. (doi:10.1095/biolreprod.103.023234)

Yang HY, Kim J, Kim SH, Choe CH \& Jang YS 2013 Pro-IL-16 is associated with $\mathrm{MHC}$ class II-mediated negative regulation of mouse resting $\mathrm{B}$ cell activation through MAP kinases, NF-kappaB and Skp2-dependent p27kip regulation. Scandinavian Journal of Immunology 77 177-186. (doi:10.1111/sji.12026)

Received 14 May 2014

Accepted 24 June 2014 